\title{
Supravaginal Hysterectomy Performed in Place of Induction of Abortion and Subsequent Steriliza- tion for Repeated Melancholia following Labours.
}

\author{
By W. S. A. Griffith, M.D.
}

THE question of the premature termination of pregnancy in mental cases is one that presents many difficulties which cannot be determined in any case by hard and fast rules.

In the first place, it is necessary to exclude those cases whose disease is an aversion to pregnancy, and whose attempts to simulate mental disease may be clever enough to gain their purpose.

We must also exclude cases in which no benefit to their mental state is likely to follow the operation.

If we succeed in excluding these two groups we are left to deal with the third group, in which we may reasonably expect that our interference will be followed by unmistakable benefit to the patient.

The case I bring before you belongs to the third group, and the point $I$ raise for discussion is the special reasons that necessitated the termination of pregnancy in the manner in which it was done.

The termination of pregnancy for any of the well recognized causes may on a single occasion be performed after due consideration without question as to the propriety of so doing, but if the woman is young and the disease chronic, as in chronic nephritis, and these mental cases, we may well hesitate to induce abortion on each recurrence of pregnancy, which may indeed mean two or three times in a year. There is therefore a group of cases in which sterilization is urgently needed as an additional method of treatment. In mental cases, of all others, it is essential that the operation should be painless, simple, safe, and of short duration, so as to diminish the mental strain on the patient as far as possible.

Induction of abortion, without an anæsthetic, means usually a period of somewhere about twenty-four hours, and then an anæsthetic may be needed to complete the emptying of the uterus, and the administration of an anæsthetic means to the patient an operation. A short time after, the operation for sterilization would have to be performed. It seemed therefore desirable, when the case to be referred to came under consideration, to combine the two in one operation by a supra-vaginal hysterectomy, leaving the ovaries, and endeavouring to leave some of the mucous membrane of the body of the uterus also. The result to the patient seems to justify this procedure under the particular circumstances of the case. 
I first saw the patient in June 1907, with Dr. Mackintosh of Hampstead. Her age was then 24. She was confined in the May previously, the labour being terminated with forceps to the large head. No mental or other complications arose until ten days after delivery. She then became listless and melancholic, with suggestions of suicide. Under Dr. Mackintosh's care she gradually recovered, and had no further trouble until her next confinement in February 1910.

Her convalescence was normal until the fourth week, when her depression returned greater, and she needed careful nursing for five months. Unfortunately, at the end of the year she again became pregnant, and I saw her with Dr. Mackintosh in January 1911. Pregnancy, it was believed, commenced about October 241910. $I$ found her to be in the third month of pregnancy, and very anxious indeed as to her possibility of going through this. She was a devoted mother to her children, and anxious to have more if she could have done so safely. Her family history was bad on both sides. It was decided to consult Dr. Percy Smith as to her mental condition. She was a bright, cheerful woman, with great self-control.

On January 20 Dr. Percy Smith saw her, and agreed that the pregnancy should be terminated, and that effective steps should be taken to prevent the recurrence of pregnancy. He also approved of the method suggested, as it appeared to involve the least suffering and the least strain. On January 271911 supra-vaginal hysterectomy was performed. Both ovaries and tubes, being healthy, were left.

With the exception of a few hours on the second day after the operation, on which there was some evidence of depression, she made an uninterrupted convalescence, and now continues in robust health. 